\title{
Prediction of rms charge radius of proton using proton-proton elastic scattering data at $\sqrt{s}=2.76 \mathrm{TeV}$
}

\author{
S. Zahra ${ }^{a}$ and B. Shafaq ${ }^{b}$ \\ ${ }^{a}$ Department of Physics, DSNT, University of Education, Lahore, Pakistan. \\ e-mail: sarwat.zahra@ue.edu.pk \\ ${ }^{b}$ CHEP, University of the Punjab, Lahore, Pakistan.
}

Received 3 October 2020; accepted 18 January 2021

\begin{abstract}
Using proton-proton elastic scattering data at $\sqrt{s}=2.76 \mathrm{TeV}$ and squared four-momentum transfer $0.36<-t<0.76(\mathrm{GeV} / \mathrm{c})^{2}$ for 13

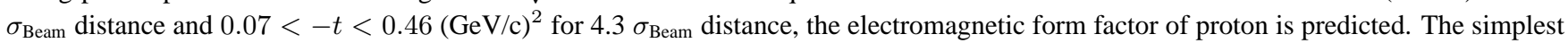
version of Chou-Yang model is employed to extract the form factor by fitting experimental data of differential cross section from TOTEM experiment (for $13 \sigma_{\text {Beam }}$ and $4.3 \sigma_{\text {Beam }}$ distance) to a single Gaussian. Root mean square charge radius of proton is calculated using this form factor and is found to be equal to $0.91 \mathrm{fm}$ and $0.90 \mathrm{fm}$, respectively. This result is in good agreement with experimental data and theoretically predicted values.
\end{abstract}

Keywords: Chou-Yang model; p-p scattering; electromagnetic form factor of proton.

DOI: https://doi.org/10.31349/RevMexFisE.67.491

\section{Introduction}

The structure of particles can be probed with the help of scattering experiments. The high energy scattering processes are now approachable present us with an opportunity to examine the hadronic structure at higher energies [1-9]. Among hadrons, the proton structure has remained a topic of interest between researchers since its discovery. Proton's radius is a prime problem in the study of its structure. The root-meansquare (rms) radius of a proton can be experimentally measured by two methods; electron proton scattering [10] and atomic spectroscopy technique. In hydrogen spectroscopy, two methods are adopted: one by using atomic hydrogen [11] and a second by using muonic hydrogen [12]; both of these methods give contradictory results, giving rise to the so-called "proton radius puzzle". There are many theoretical approaches to find out the rms radius of proton, including MIT Bag model [13], self-consistent model [14], by using Lattice QCD [15-17], etc.

The form factor also plays a dynamic role in the study of hadronic structure. It is related to the distribution of matter inside a hadron. Theories claiming to explain the structure of hadrons must be able to calculate their form factors from first principles. Continuous efforts of decades led the researchers to obtain the form factors of proton from different calculation schemes, as discussed in [18-22]. Experimentally, the magnitude of the form factor is determined by the ratio of the measured cross-section to the Mott cross-section: $(d \sigma / d \Omega)_{\exp }=(d \sigma / d \Omega)_{\text {Mott }}^{*} \cdot\left|F\left(q^{2}\right)\right|^{2}$. One therefore measures the cross-section for a fixed beam energy at various angles (and thus different values of $|q|$ ) and divides by the calculated Mott cross-section.

In this work, proton-proton elastic scattering data from TOTEM experiment [9] is used to calculate the proton form factor employing the simplest version of Chou-Yang model. The Chou-Yang model $[23,24]$ is a geometrical model. In this model two hadrons are considered to be scattering elastically and are supposed to be translucent objects passing through each other without attenuation. The differential cross section $(d \sigma / d t)$ and total cross-section $(\sigma)$ are the two measuring quantities involved in such processes. Let us consider two hadrons $A$ and $B$, scattering elastically $(A+B \rightarrow A+B)$. Let $a_{A B}(t)$ represent the asymptotic scattering amplitude. Here we are interested in a case, where Gaussian (in $t$ ) could be used to approximate the differential cross section. In this situation the differential cross section is written as

$$
\frac{d \sigma}{d t}=\alpha e^{\beta t}
$$

Hadron's radius is associated to the form factor by the relation $F_{A}(t)=1-\left(1 / 6 \hbar^{2}\right) t\left\langle r^{2}\right\rangle$. The product of both form factors $\left(F_{A}(t)\right.$ and $\left.F_{B}(t)\right)$ of two scattering hadrons $A$ and $B$ is given as:

$$
\begin{aligned}
F_{A}(t) F_{B}(t) & =\text { constant } \\
& \times \sum_{n=1}^{\infty} \frac{1}{n}\left(\frac{\alpha}{\pi}\right)^{n / 2}\left(\frac{1}{\beta}\right)^{n} \frac{\beta}{n} e^{\beta t / 2 n},
\end{aligned}
$$

(for details see Ref. [25]). This relation is very useful for finding out the form factor of scattering hadrons. Many form factors of proton were suggested by researchers at lower values of $\sqrt{s}$ [26-28].

\section{Calculations}

In this work recent data of elastic proton-proton scattering at $\sqrt{s}=2.76 \mathrm{TeV}$ from TOTEM experiment [9] is used. Al- 


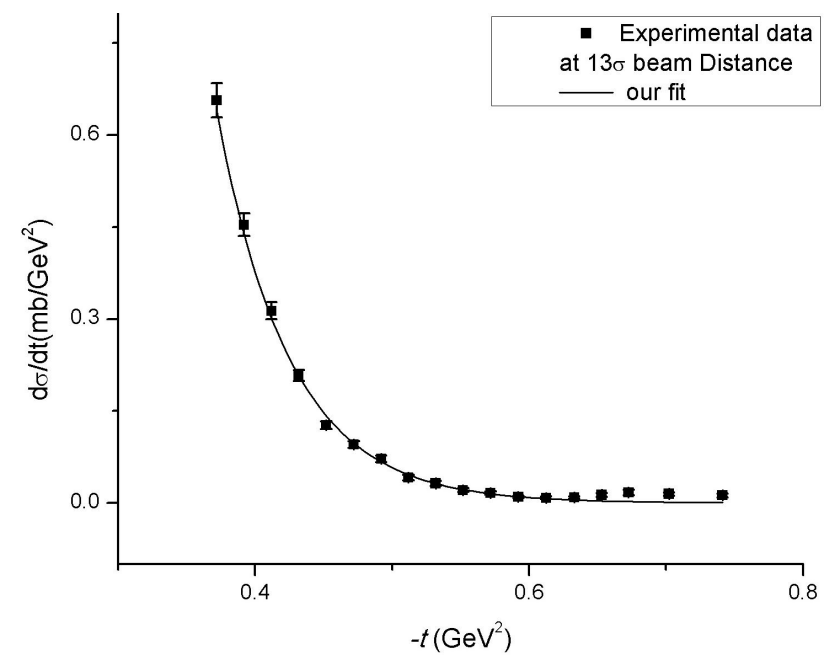

FIGURE 1. Fitting of differential cross section data of protonproton elastic scattering at $\sqrt{s}=2.76 \mathrm{TeV}$ ( for $13 \sigma_{\text {Beam }}$ distance) to a single Gaussian.

though the same procedure has been adopted in [18] for finding out form factor and rms radius of proton by using the data from TOTEM at $\sqrt{s}=8 \mathrm{TeV}$. Here elastic protonproton data of two different kinematical regions is analyzed at $\sqrt{s}=2.76 \mathrm{TeV}$ which would be highly beneficial for getting precise results. The experimental setup of TOTEM experiment is explained in Ref. [9], where one of the data sets of differential cross section has been obtained by placing Roman Pot detectors at 13 times the transverse beam size $\left(\sigma_{\text {Beam }}\right)$. This setup allowed to measure elastic differential cross section at $t=0.36 \mathrm{GeV}^{2}$ to $0.74 \mathrm{GeV}^{2}$. The second data set was obtained by inserting Roman Pot detectors at 4.3 times the transverse beam size and measured elastic differential cross section at $t=0.07 \mathrm{GeV}^{2}$ to $0.45 \mathrm{GeV}^{2}$. The differential cross section data plotted against $-t$ for $13 \sigma_{\text {Beam }}$ and $4.3 \sigma_{\text {Beam }}$ distance, and fitted to a single Gaussian, shown in Fig. 1 and 2, respectively.

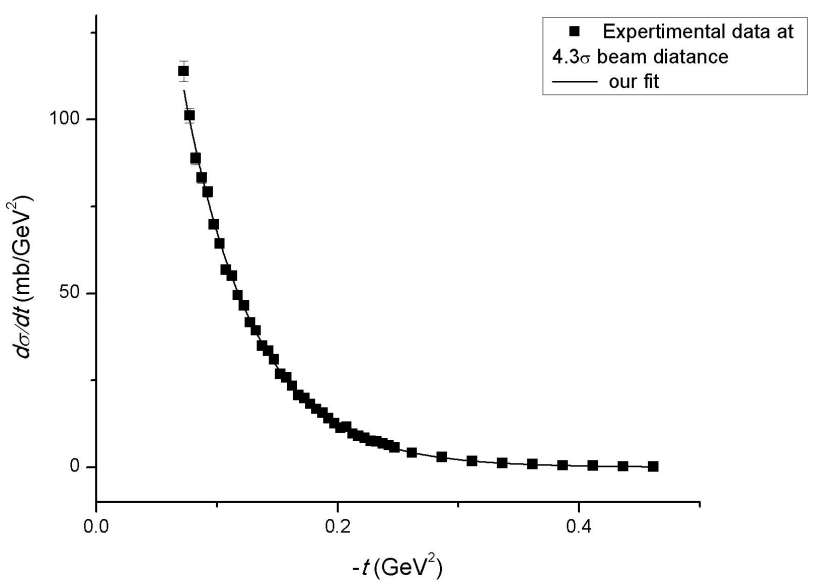

FIGURE 2. Fitting of differential cross section data of protonproton elastic scattering at $\sqrt{s}=2.76 \mathrm{TeV}$ (for $4.3 \sigma_{\text {Beam }}$ distance) to a single Gaussian.
TABLE I. Fitted parameters for two set of data, i.e., for $13 \sigma_{\text {Beam }}$ and $4.3 \sigma_{\text {Beam }}$ distance

\begin{tabular}{cccc}
\hline$j$ & $\begin{array}{c}\alpha_{j} \\
\left(\mathrm{mb}^{-1} / \mathrm{GeV} / \mathrm{c}\right)\end{array}$ & $\beta_{j}(\mathrm{GeV})^{-2}$ & $\begin{array}{c}\text { Adj. R-squared } \\
\text { for fit }\end{array}$ \\
\hline 1 & $683.06 \pm 228.29$ & $-18.75 \pm 0.76886$ & 0.96907 \\
2 & $375.62 \pm 4.09935$ & $-17.15 \pm 0.06806$ & 0.99914 \\
\hline
\end{tabular}

TABLE II. Computed values of $a_{j i}$ and $b_{j i}$.

\begin{tabular}{ccccc}
\hline & \multicolumn{2}{c}{ For 13} & $\sigma_{\text {Beam }}$ & \multicolumn{2}{c}{ For 4.3} & $\sigma_{\text {Beam }}$ \\
& \multicolumn{2}{c}{ distance $j=1$} & \multicolumn{2}{c}{ distance $j=2$} \\
\hline$i$ & $a_{j i}$ & $b_{j i}$ & $a_{j i}$ & $b_{j i}$ \\
\hline 1 & -2.898290 & -18.75452 & -1.742644 & -17.1527 \\
2 & 14.74531 & -9.37726 & 10.93454 & 10.93454 \\
\hline
\end{tabular}

TABLE III. Computed values of $\delta_{j}$.

\begin{tabular}{cc}
\hline & $\delta_{j}$ \\
\hline For $13 \sigma_{\text {Beam }}$ Distance & 0.2905 \\
For $4.3 \sigma_{\text {Beam }}$ Distance & 0.3298 \\
\hline
\end{tabular}

The most appropriate values of $\alpha_{1}, \beta_{1}$ and $\alpha_{2}, \beta_{2}$ are found. Where $\alpha_{1}, \beta_{1}$ are the fitted parameters for differential cross section data at $13 \sigma_{\text {Beam }}$ distance and are for 4.3 $\sigma_{\text {Beam }}$ distance. These fitted parameters given in Table I.

The measure of goodness of fit is determined by Rsquare, given in Table I. Which shows that our fit is $100 \%$ successful in both set of data. These values of $\alpha$ and $\beta$ are used in Eq. (2) directly, and a computer program is used to solve this equation for finite values of $n$. The square of form factors of proton is found to be equal to

$$
\left(F_{j p}(t)\right)^{2}=\delta_{j} \sum_{i=1}^{2} a_{j i} e^{b j_{i} t} .
$$

Here $j=1$ and 2 for differential cross section data at 13 $\sigma_{\text {Beam }}$ and $4.3 \sigma_{\text {Beam }}$ distance respectively. $\delta_{j}$ is the normalization constant. Computed values are given in Table II and Table III.

Using electromagnetic form factor from Eq. (3) we can easily compute rms charge radius of proton by using following relation $\left\langle r^{2}\right\rangle=\left.6 \hbar^{2}(d F(t) / d t)\right|_{t=0}$. We have therefore computed $\left\langle r_{p}\right\rangle=0.91 \mathrm{fm}$ and $\left\langle r_{p}\right\rangle=0.90 \mathrm{fm}$ for $13 \sigma_{\text {Beam }}$ and $4.3 \sigma_{\text {Beam }}$ distance respectively which is in good agreement with the experiment $\left\langle r_{p}\right\rangle=0.84 \pm 0.00039 \mathrm{fm}$ [29].

\section{Discussion}

The Chou Yang model is successful in its predictions for elastic scattering process at higher as well as the lower values of $\sqrt{s}$. The proton electromagnetic form factor is obtained at low squared momemtm transfer, i.e., $0.36<-t<0.76(\mathrm{GeV} / \mathrm{c})^{2}$ 


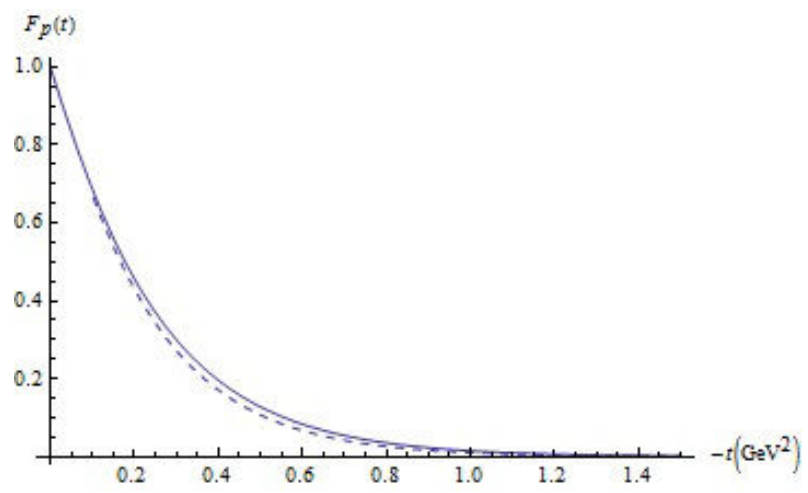

FIGURE 3. Form factor of proton predicted (for $13 \sigma_{\text {Beam }}$ distance and $4.3 \sigma_{\text {Beam }}$ distance).

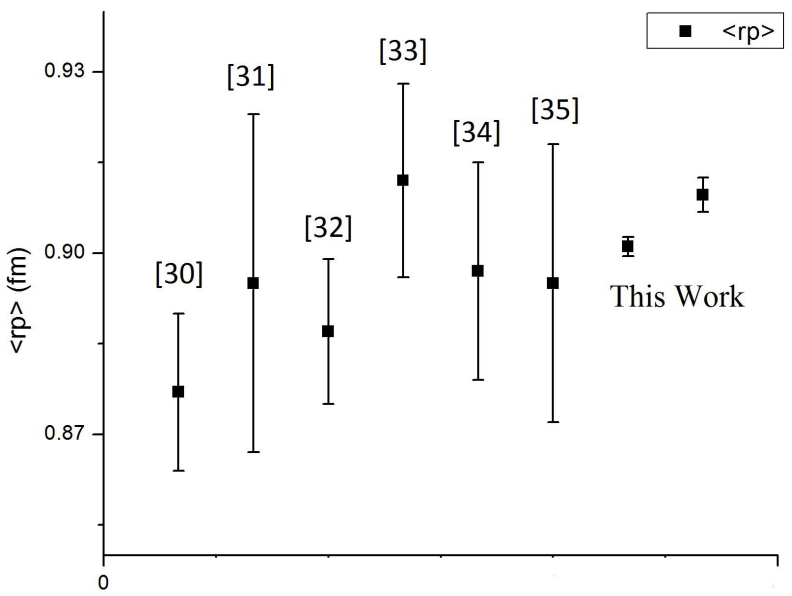

FIGURE 4. Comparison of calculated rms radii. and $0.07<-t<0.46(\mathrm{GeV} / \mathrm{c})^{2}$, but high centre-of-mass energy i.e $\sqrt{s}=2.76 \mathrm{TeV}$. Figures 1 and 2 show the points of the experimental data from TOTEM [9] represented by squares, whereas our fit is drawn with a solid line. We have obtained the most suitable fit. Figure 3 shows the comparison plot of our predicted form factor for both sets of data, where the dotted line shows the form factor for $13 \sigma_{\text {Beam }}$ distance data and solid line shows the form factor predicted for $4.3 \sigma_{\text {Beam }}$ distance. The novel aspect of this work is that the simplest method is employed and our calculated rms charge radius of proton that agree well with experiment and theory. A comparison of calculated values is given in Fig. 4.
1. G. Antchev et al., Proton-proton elastic scattering at the LHC energy of $\sqrt{s}=7 \mathrm{TeV}$, EPL. 95 (2011) 41001. 10.1209/ $0295-5075 / 95 / 41001$.

2. G. Antchev et al., First measurement of the total protonproton cross-section at the LHC energy of $\sqrt{s}=7 \mathrm{TeV}$, EPL. 96 (2011) 21002. https://doi.org/10.1209/ $0295-5075 / 96 / 21002$.

3. G. Antchev et al., Luminosity-Independent Measurement of the Proton-Proton Total Cross Section at $\sqrt{s}=8 \mathrm{TeV}$, Phys. Rev Letts. 111 (2013) 012001. https: //doi.org/10.1103/ PhysRevLett.111.012001.

4. TOTEM Collaboration, Evidence for non-exponential elastic proton-proton differential cross-section at low $|t|$ and $\sqrt{s}=8$ TeV by TOTEM, Nucl. Phys. B. 899 (2015) 527. https: //doi.org/10.1016/j.nuclphysb.2015.08.010

5. G. Antchev, et al., Measurement of elastic pp scattering at $\sqrt{s}=8 \mathrm{TeV}$ in the Coulomb-nuclear interference region: determination of the $\rho$-parameter and the total crosssection, EPJC. 76 (2016) 661. https://doi.org/10. 1140/epjc/s10052-016-4399-8.
6. G. Antchev, et al., First determination of the $\rho$-parameter at $\sqrt{s}=13 \mathrm{TeV}$ : probing the existence of a colourless C-odd three-gluon compound state, EPJC. 79 (2019) 785. https: //doi.org/10.1140/epjc/s10052-019-7223-4.

7. G. Antchev et al., Elastic differential cross-section measurement at root $s=13 \mathrm{TeV}$ by TOTEM, EPJC. 79 (2019) 861. https://doi.org/10.1140/epjc/ s10052-019-7346-7

8. G. Antchev, et al., First measurement of elastic, inelastic and total cross-section at $\sqrt{s}=13 \mathrm{TeV}$ by TOTEM and overview of cross-section data at LHC energies, EPJC. 79 (2019) 103. https://doi.org/10.1140/epjc/ s10052-019-6567-0

9. G. Antchev et al., Elastic differential cross-section d sigma/dt at root $s=2.76 \mathrm{TeV}$ and implications on the existence of a colourless C-odd three-gluon compound state, EPJC. 80 (2020) 91. https://doi.org/10.1140/ epjc/s10052-020-7654-y.

10. J. C. Bernauer, et al., Electric and magnetic form factors of the proton, Phys. Rev. C, 90 (2014) 015206. https: / / doi. org/10.1103/PhysRevC.90.015206 
11. P. J. Mohr, B. N. Taylor, and D. B. Newell, CODATA recommended values of the fundamental physical constants: 2006, $J$ Phys Chem Ref Data. 37 (2008) 1187. https: //doi.org/ $10.1063 / 1.2844785$.

12. A. Antognini, et al., Proton Structure from the Measurement of 2S-2P Transition Frequencies of Muonic Hydrogen. Science 339 (2013) 417. 10.1126/science.1230016.

13. P. K. Chatley, C. P. Singh, and M. P. Khanna, Charge radii of proton and $\mathrm{M} 1$ radiative transitions of hadrons in a bag model with variable bag pressure. Phys. Rev. D. 29 (1984) 96. https://doi.org/10.1103/PhysRevD.29.96.

14. S.G. Fedosin, The radius of the proton in the self-consistent model. Had. Jour. 35 (2012) 349.

15. N. Hasan et al., Computing the nucleon charge and axial radii directly at $Q^{2}=0$ in lattice QCD, Phys. Rev. D. 97 (2018) 034504. 10.1103/PhysRevD.97.034504

16. F. M. Stokes, W. Kamleh, and D. B. Leinweber, Elastic form factors of nucleon excitations in lattice QCD, Phys. Rev. D. 102 (2020) 014507. https://doi.org/10.1103/ PhysRevD.102.014507

17. Y. C. Jang, R. Gupta, H. W. Lin, B. Yoon, T. Bhattacharya, and PNDME Collaboration, Nucleon electromagnetic form factors in the continuum limit from $(2+1+1)$-flavor lattice QCD, Phys. Rev. D. 101 (2020) 014507.https://doi.org/10. 1103/PhysRevD.101.014507

18. S. Zahra, and H. Rashid, Predictions of the Chou-Yang Model for pp Scattering at $\sqrt{s}=8 \mathrm{TeV}$, Chin. Phys. Letts. 36 (2019) 061201.10.1088/0256-307X/36/6/061201

19. C. A. Dominguez, Electromagnetic form factors of hadrons in quantum field theories, AIP Conference Proceedings (Vol. 1056, No. 1, 23-30), (2008) American Institute of Physics. https://doi.org/10.1063/1.3013047

20. O. D. Dalkarov, P. A. Khakhulin, and A. Y. Voronin, On the electromagnetic form factors of hadrons in the time-like region near threshold, Nucl. Phys. A. 833 (2010) $10410.1016 / j$. nuclphysa.2009.11.015

21. D. B. Leinweber, et al., Strange electric form factor of the proton, Phys. Rev. Letts. 97 (2006) 022001, https://doi. org/10.1103/PhysRevLett.97.022001

22. J. J. Murphy, I. I., Shin, Y. M. and D. M. Skopik, Proton form factor from 0.15 to 0.79 fm2, Phys. Rev. C. 9 (1974) 2125. https://doi.org/10.1103/PhysRevC.9.2125
23. T. T. Chou, and C. N. Yang, Model of elastic high-energy scattering, Phys. Rev. 170 (1968) 1591. https://doi.org/ 10.1103/PhysRev.170.1591

24. A. W. Chao, and C. N. Yang, Opaqueness of pp collisions from 30 to $1500 \mathrm{GeV} / \mathrm{c}$, Phys. Rev. D. 8 (1973) 2063. https : //doi.org/10.1103/PhysRevD.8.2063

25. S.-Y. Lo., Geometrical Pictures in Hadronic Collisions: A Reprint Volume, (World Scientific, 1987). Chap. 4, p. 37

26. L. Durand III, and R. Lipes, Diffraction Model for High-Energy pp Scattering, Phys. Rev. Letts. 20 (1968) 637. https:// doi.org/10.1103/PhysRevLett.20.637

27. J. G. Rutherglen, in Proceedings of the 4th International Symposium on Electron and Photon Interactions at High Energies, Liverpool, (1969) Sept. 14-20, Ed. by D. W. Braben (Liverpool Daresbury, Univ. of Glasgow), p. 163.

28. M. Saleem, and I. A. Azhar, Generalized Chou-Yang Model for $p(\bar{p}) p$ and $\Lambda(\bar{\Lambda}) \mathrm{p}$ Elastic Scattering at High Energies, EPL. 6 (1988) 201.10.1209/0295-5075/6/3/003

29. P. A. Zyla et al. (Particle Data Group), Prog. Theor. Exp. Phys. 2020 (2020) 083C01,

30. H. Fleurbaey, New measurement of the $1 \mathrm{~S}-3 \mathrm{~S}$ transition frequency of hydrogen: contribution to the proton charge radius puzzle, Phys. Rev. Letts. 120 (2018) 183001.10.1103/ PhysRevLett.120.183001

31. G. Lee, J.R. Arrington, and R.J. Hill, Extraction of the proton radius from electron-proton scattering data, Phys. Rev. D. 92 (2015) 013013. https : //doi.org/10.1103/ PhysRevD.92.013013

32. Sick, Ingo. Proton charge radius from electron scattering, Atoms 6 (2018) 2. https://doi.org/10.3390/ atoms 6010002

33. D. Borisyuk, Proton charge and magnetic rms radii from the elastic ep scattering data, Nucl. Phys. A. 843 (2010) 59. https://doi.org/10.1016/j.nuclphysa.2010. 05.054

34. P.G. Blunden, and I. Sick, Proton radii and two-photon exchange, Phys. Rev. C. 72 (2005) 057601. https://doi. org/10.1103/PhysRevC.72.057601

35. I. Sick, On the rms-radius of the proton, Phys. Letts. B. 576 (2003) 62, https://doi.org/10.1016/j. physletb.2003.09.092 